questionable, and others are unfortunately divorced from the caveats and cautions made explicit by their originators. But most are succinct and informative, with a number of the maps being original and worthy of being brought to the attention of researchers in this field.

The final third of this book comprises six appendices detailing, respectively, the phyla of living organisms, important food crops, domestic livestock, recent vertebrate extinctions, biodiversity at country level, and important areas for freshwater biodiversity. Much of this material is rapidly becoming staple for volumes on biodiversity, and is available elsewhere, so one has to question the value of its repeated inclusion (accepting that some of it receives frequent revision). This, of course, rests on who the readership is likely to be. There are many people around the world who would learn much that is of importance from reading this book, including educators, politicians, policy-makers and the general public. But it must do more than simply get onto their shelves, it needs to be well thumbed. If the authors and publishers can achieve this, they will have done all 6 billion of us a great service.

Kevin J. Gaston is in the Department of Animal and Plant Sciences, University of Sheffield, Sheffield S10 2TN, UK.

\section{Mathematics off the main line}

\section{Mathematics Elsewhere: An Exploration of Ideas Across Cultures}

by Marcia Ascher

Princeton University Press: 2002. 224 pp.

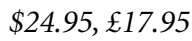

\section{John O'Connor}

Mathematicians like to trace the ancestry of their subject back to the Renaissance and then, with a bit of disruption due to the Dark Ages (and some help from Islamic and Indian mathematicians), back to the Ancient Greeks, and perhaps even earlier to the Babylonians, Egyptians or the oldest Middle Eastern civilizations that we know of. More recently, however, we have become aware that many of the important ideas that we have placed on this main line of development were discovered independently in a variety of other societies.

Marcia Ascher's latest book provides yet more evidence that mathematical ideas that Western mathematicians have regarded as recent sophisticated constructs also have roots in geographically diverse cultures. She considers several different areas where such independent development has taken place.

Calendars offer an insight into how vari-
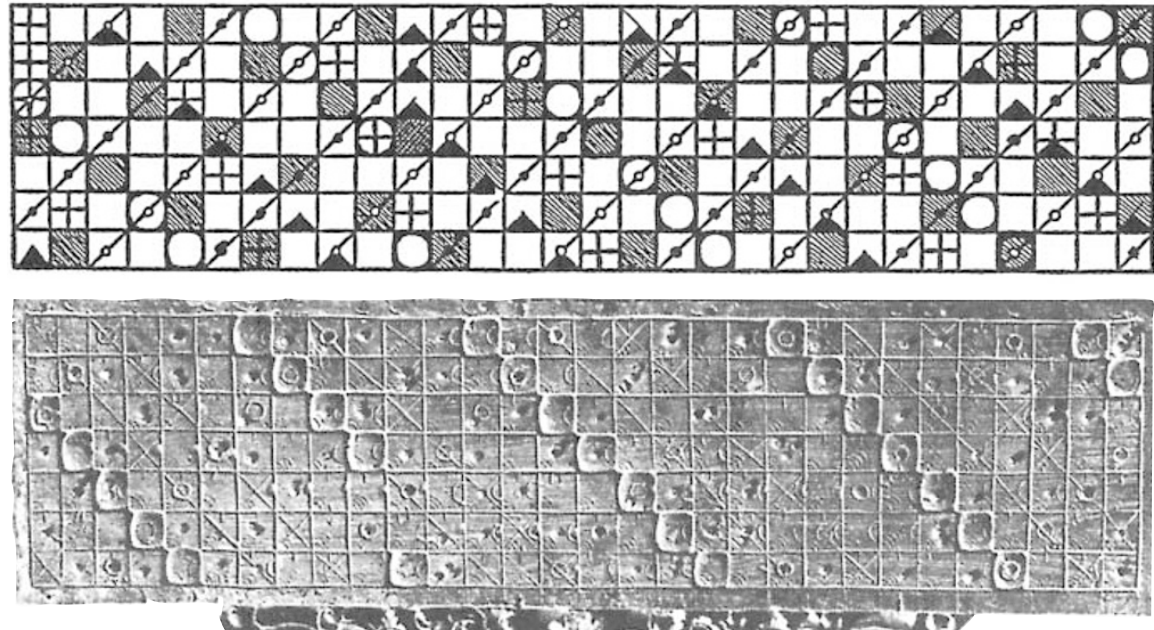

Marking time: the

Balinese calendar, here represented on paper (top) and wooden tika requires complex calculations.

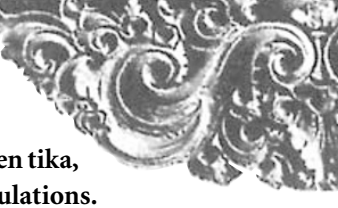

ous societies have attempted to resolve the problems associated with the different length cycles of Sun, Moon and seasons. We feel at home with our Western calendar in its present gregorian variant, and it is only when we contemplate something like the rule for locating Easter that we realize just how much calculation it requires. The mathematician Carl Friedrich Gauss developed the system that we now use for modular arithmetic to handle the problems associated with calculating Easter. More recently, the version of the formula printed in The Book of Common Prayer was one of the influences that turned Bertrand Russell towards mathematics.

In Mathematics Elsewhere, Ascher considers a variety of other calendars: two from South Pacific cultures, as well as the Jewish, Mayan and Balinese versions. All of these lead to extremely intricate calculation problems. The Balinese calendar, for example, has ten weeks of different length running simultaneously, each with its own labelling system for days. The problem of moving backwards and forwards between these different systems would tax the mentalarithmetic capacity even of those of us raised to calculate in pounds, shillings and pence, let alone those from the decimalcurrency and calculator age. As the author points out: "Some of the mathematical ideas and questions are restricted to specialists, but, nevertheless, the logic of interlocked cycles is pervasive in the cultures."

Other 'modern' ideas crop up in unlikely places in tribal cultures. The divination rituals practised in the Caroline archipelago in the Pacific, in some areas of Nigeria and in the island of Madagascar have common elements related to the mathematics of boolean algebra, and involve algorithms only recognized as such by twentieth-century mathematicians. Most of these processes operate by 